\title{
Obtaining representable coimplications from aggregation and dual operators
}

\author{
Renata H. S. Reiser ${ }^{1}$ Benjamín C. Bedregal $^{2}$ \\ ${ }^{1}$ CDTEC, UFPEL, CP 354, Campus Universitário, 96010-900 Pelotas, Brazil \\ ${ }^{2}$ DIMAP, UFRN, Campus Universitário, 59072-970 Natal, Brazil
}

\begin{abstract}
The aim of this work is to study $N$-dual structures of representable implications generated from a set $M$ of aggregation functions and pairs of mutual dual functions, founded on the isomorphism between the unit interval and special instances of the Goguen's $\mathcal{L}$-fuzzy sets. We discuss under which conditions representable functions preserve the main properties of their $N$-dual interval approach.
\end{abstract}

Keywords: Interval-valued fuzzy logic, representable coimplications, $N$-dual interval-valued coimplications.

\section{Introduction}

According to [29], Goguen's $\mathcal{L}$-fuzzy sets have been the source of various generalizations in interval-valued fuzzy set theory. In [18], fuzzy sets with rough sets were combined, despite their distinct starting points often related to sets with smooth boundaries and gradual notions of knowledge. Since [39, 42, 43] and [26], such and many other approaches, see also [18] and [34], have been studied, dealing with information granulation, reducing the uncertainty related to the imprecision from the membership grades.

Introduced by Atanassov [1], intuitionistic fuzzy sets were defined as a pair of membership functions indicating both the degrees of membership and of nonmembership. The work in [10] states that an intuitionistic fuzzy implication operator recovers J. Fodor's definition of a fuzzy implication operator when the sets are fuzzy and also generalizes the Atanassov's operator [2]. In $[12,13]$ and [14], the definition of fuzzy connectives are undertaken by such theories.

The main contribution of this paper is the introduction of an $N$-dual structure concept related to $\left(I, I_{N}, M\right)$-representable implication generated from a set $M$ of aggregation functions and mutual dual fuzzy implications $I$ and $I_{N}$, based on the isomorphism between $\mathbb{U}^{2}$ and $\mathcal{L}^{*}$ by mapping the notions presented in [10, Definition 3] to the canonical representation [5]. We discuss under which conditions such representable implications preserve the main properties of interval implications, showing the applicability of $N$-dual interval approach for representable implications.

The paper is organized as follows. Section 2 discusses the notions of interval representations of real functions and presents definitions and main results concerned with duality relationship, fuzzy negations and aggregation operators. The basic concepts of fuzzy (co)implications are studied in Section 3 followed by Section 4, where firstly, the main results related to the interval extensions of fuzzy (co)implications introduced in previous works $[5,6]$ are presented. Thus, in Section 5, we focus on interval-valued (co)implications generated by aggregation functions and by mutual dual fuzzy (co)implications, proving that several analogous properties of fuzzy (co)implications are also held for such representable (co)implications. In Section 6, we conclude by pointing out the main results of this paper, the ongoing work and some final remarks.

\section{Interval Representations}

Consider the real unit interval $U=[0,1] \subseteq \mathbb{R}$ and let $\mathbb{U}$ be the set of subintervals of $U$, that is, $\mathbb{U}=\{X=$ $[a, b] \mid 0 \leq a \leq b \leq 1\}$. The interval set has two projections $l, r: \mathbb{U} \rightarrow U$, defined by $l(X)=l([a, b])=$ $a$ and $r(X)=r([a, b])=b$ and denoted by $\underline{X}$ and $\bar{X}$, respectively.

The partial orders that are considered in this paper are the inclusion relation and the component-wise KulischMiranker order (also called product order), defined by:

$$
\forall X, Y \in \mathbb{U}: X \leq Y \Leftrightarrow \underline{X} \leq \underline{Y \wedge} \bar{X} \leq \bar{Y} .
$$

An interval $X \in \mathbb{U}$ is said to be an interval representation of a real number $\alpha$ if $\alpha \in X$. Considering two interval representations $X$ and $Y$ of a real number $\alpha$, $X$ is said to be a better representation of $\alpha$ than $Y$ if $X \subseteq Y$. This notion can be easily extended for tuples of $n$ intervals $\vec{X}=\left(X_{1}, \ldots, X_{n}\right)$.

Definition 1 A function $F: \mathbb{U}^{n} \longrightarrow \mathbb{U}$ is an interval representation of a function $f: U^{n} \longrightarrow U$ if, for each $\vec{X} \in \mathbb{U}^{n}$ and $\vec{x} \in \vec{X}, f(\vec{x}) \in F(\vec{X})$ [40]. ${ }^{1}$

Definition 2 Let $F: \mathbb{U}^{n} \longrightarrow \mathbb{U}$ and $G: \mathbb{U}^{n} \longrightarrow \mathbb{U}$ be two interval representations of the function $f: U^{n} \longrightarrow$ $U$. $F$ is a better interval representation of $f$ than $G$, denoted by $G \sqsubseteq F$, if, for each $\vec{X} \in \mathbb{U}^{n}$, the inclusion $F(\vec{X}) \subseteq G(\vec{X})$ holds.

Definition 3 For each real function $f: U^{n} \longrightarrow U$, the interval function $\widehat{f}: \mathbb{U}^{n} \longrightarrow \mathbb{U}$, defined by

$$
\widehat{f}(\vec{X})=[\inf \{f(\vec{x}) \mid \vec{x} \in \vec{X}\}, \sup \{f(\vec{x}) \mid \vec{x} \in \vec{X}\}],
$$

is called the canonical representation of $f[40]^{2}$

\footnotetext{
${ }^{1}$ Notice that the concept of interval representation is different from interval extension and natural extension. [35, page 21]

${ }^{2}$ Notice that $\widehat{f}$ is the interval hull of the range of $f$.
} 
The interval function $\widehat{f}$ is well defined and for any other interval representation $F$ of $f, F \sqsubseteq \widehat{f}$. The interval function $\widehat{f}$ returns an interval that is narrower than any other interval representation of $f$. Notice that if $f$ is continuous, in the usual sense, then for each $\vec{X} \in \mathbb{U}^{n}$, $\widehat{f}(\vec{X})=f(\vec{X})$, where $f(\vec{X})=\{f(\vec{x}) \mid \vec{x} \in \vec{X}\}$.

There are several possible notions of continuity for interval functions (see, e.g., [40]). In this paper we will take into consideration the following notions of continuity for interval functions: (i) Moore continuity, considering the metric $d(X, Y)=\max (|\underline{X}-\underline{Y}|,|\bar{X}-\bar{Y}|)$ defined over $\mathbb{U}$; (ii) Scott continuity, considering the set $\mathbb{U}$ with reverse inclusion order as a continuous domain and a function $f:(\mathbb{U}, \supseteq) \rightarrow(\mathbb{U}, \supseteq)$ as monotonic and preserving the least upper bound of directed sets [25].

\subsection{Interval Fuzzy Negations}

A function $N: U \rightarrow U$ is a fuzzy negation if

N1 $: N(0)=1$ and $N(1)=0$;

N2 : If $x \geq y$ then $N(x) \leq N(y), \forall x, y \in U$.

Fuzzy negations satisfying the involutive property given below are called strong fuzzy negations $[9,28]$ :

N3 : $N(N(x))=x, \forall x \in U$.

$\mathbb{N}: \mathbb{U} \longrightarrow \mathbb{U}$ is an interval fuzzy negation if, for all $X, Y$ in $\mathbb{U},[1,1]=\mathbf{1}$ and $[0,0]=\mathbf{0}$, the properties hold:

$\mathbb{N} \mathbf{1}: \mathbb{N}(\mathbf{0})=\mathbf{1}$ and $\mathbb{N}(\mathbf{1})=\mathbf{0}$;

N2a $:$ If $X \leq Y$ then $\mathbb{N}(Y) \leq \mathbb{N}(X) ;$

$\mathbb{N}$ 2b : If $X \subseteq Y$ then $\mathbb{N}(X) \subseteq \mathbb{N}(Y)$.

If $\mathbb{N}$ also satisfies $\mathbb{N} 3$, then $\mathbb{N}$ is a strong interval-valued negation (SIVN):

$\mathbb{N} \mathbf{3}: \mathbb{N}(\mathbb{N}(X))=X$ (involutive property).

Notice that in [8] the definition of interval fuzzy negation is required to be a monotonic inclusion function which agrees with the n-representable interval-valued negation in [15, 17] and [16]. But in the case of a strong interval fuzzy negation both definitions coincide.

A typical example of a strong interval fuzzy negation is $\mathbb{N}_{S}: \mathbb{U} \rightarrow \mathbb{U}$, defined by $\mathbb{N}_{S}(X)=[1,1]-X$, i.e. $\mathbb{N}_{S}(X)=[1-\bar{X}, 1-\underline{X}] . \mathbb{N}_{S}$ is the interval version of the Zadeh fuzzy negation $\left(N_{Z}(x)=1-x\right)$.

The interval function $\widehat{N}: \mathbb{U} \rightarrow \mathbb{U}$ of a negation $N$ : $U \longrightarrow U$ can be given by $\widehat{N}(X)=[N(\bar{X}), N(\underline{X})]$.

Definition 4 Let $N$ be a strong fuzzy negation on $U$ and $f: U^{n} \longrightarrow U$ be a real function. The $N$-dual function of $f$ is given by the expression:

$$
f_{N}\left(x_{1}, \ldots, x_{n}\right)=N\left(f\left(N\left(x_{1}\right), \ldots, N\left(x_{n}\right)\right)\right) .
$$

Notice that, because $N$ is involutive, $\left(f_{N}\right)_{N}=f$, that is the $N$-dual function of $f_{N}$ coincides with $f$. In addition, $f=f_{N}$ it is clear that $f$ is a self-dual function.
Definition 5 Let $\mathbb{N}: \mathbb{U} \rightarrow \mathbb{U}$ be a strong interval fuzzy negation on $\mathbb{U}$ and $F: \mathbb{U}^{n} \longrightarrow \mathbb{U}$ be an interval function. The $\mathbb{N}$-dual interval function of an interval function $F$ is given by the expression:

$$
F_{\mathbb{N}}\left(X_{1}, \ldots, X_{n}\right)=\mathbb{N}\left(F\left(\mathbb{N}\left(X_{1}\right), \ldots, \mathbb{N}\left(X_{n}\right)\right)\right) .
$$

Notice that, because $\mathbb{N}$ is involutive, $\left(F_{\mathbb{N}}\right)_{\mathbb{N}}=F$. When $\mathbb{N}=\mathbb{N}_{S}$ then $\mathbb{F}$ and $\mathbb{F}_{\mathbb{N}_{S}}$ are called mutual $\mathbb{N}_{S}-$ dual interval functions [33].

\section{Fuzzy (co)implications}

Coimplication functions are conceived as $N$-dual structures of implication functions extending the classical (co)implications [19, 20, 21, 9, 32] and [3]:

Definition 6 [41, Section 3.1] The binary operator $(J) I:[0,1]^{2} \rightarrow[0,1]$ is a fuzzy (co)implication if it satisfies the boundary conditions:

$$
\begin{aligned}
& \text { J1 : } J(1,1)=J(1,0)=J(0,0)=0 \text { and } J(0,1)=1 ; \\
& \text { I1 } I(1,1)=I(0,1)=I(0,0)=1 \text { and } I(1,0)=0 .
\end{aligned}
$$

Since Definition 6 imposes a very weak condition for a binary function to be a fuzzy (co)implication, several other extra properties are considered in the literature in addition to (J1) I1. The most used ones are listed below:

$$
\begin{aligned}
& \mathbf{J} 2: x \leq z \rightarrow J(x, y) \geq J(z, y) ; \mathbf{I} 2: x \leq z \rightarrow I(x, y) \geq I(z, y) \text {; } \\
& \text { J3: } y \leq z \rightarrow J(x, y) \leq J(x, z) \text {; I3: } y \leq z \rightarrow I(x, y) \leq I(x, z) \text {; } \\
& \mathbf{J} 4: J(1, y)=0 ; \quad \mathbf{I} 4: I(0, y)=1 ; \\
& \text { J5: } J(x, 0)=0 ; \quad \mathbf{I} 5: I(x, 1)=1 \text {; } \\
& \text { J6: } J(0, y)=y ; \quad \mathbf{I} 6: I(1, y)=y \text {; } \\
& \mathbf{J} 7: J(x, J(y, z)=J(y, J(x, z) ; \mathbf{I} 7: I(x, I(y, z)=I(y, I(x, z) ; \\
& \text { J8: } J(x, y)=0 \leftrightarrow x \geq y ; \quad \mathbf{I} 8: I(x, y)=1 \leftrightarrow x \leq y ; \\
& \text { J9: } N_{J}(x)=J(x, 1) \text { is SFN; } \mathbf{I} \text { 9: } N_{I}(x)=I(x, 0) \text { is SFN; } \\
& \text { J10: } J(x, y) \leq y ; \quad \text { I10: } I(x, y) \geq y \text {; } \\
& \text { J11: } J(x, x)=0 ; \quad \text { I11: } I(x, x)=1 \text {; } \\
& \text { J12: } J(x, y)=J(N(y), N(x) ; \mathbf{I} 12: I(x, y)=I(N(y), N(x) \text {; } \\
& \mathbf{J} 13: x<1 \rightarrow J(x, 1)>0 ; \quad \mathbf{I} 13: x>0 \rightarrow I(x, 0)<1 \\
& \mathbf{J} 14: y>0 \rightarrow J(0, y)>0 ; \quad \mathbf{I} 14: y<1 \rightarrow I(1, y)<1 \\
& \text { J15: } J \text { is continuous; } \quad \text { I15: } I \text { is continuous } \\
& \text { J16: } J(x, y)=J(x, J(x, y)) \text {; I16: } I(x, y)=I(x, I(x, y)) \text {; } \\
& \text { J17: } J(x, J(y, x))=0 ; \quad \text { I17: } I(x, I(y, x))=1 \text {; } \\
& \text { J18: } J(x, J(y, z)) \geq \quad \text { I18: } I(x, I(y, z)) \leq \\
& J(J(x, y), J(x, z)) ; \quad I(I(x, y), I(x, z)) ; \\
& \text { J19: } J(N(x), x)=x ; \quad \text { I19: } I(x, N(x))=N(x) \text {; }
\end{aligned}
$$

Prop. 7 states how an implication gives rise to a coimplication and vice-versa, see also [30, 31, 38] and [4].

Proposition 7 Let $N$ be a strong fuzzy negation. If a function $(J) I: U^{2} \rightarrow U$ is a (co)implication then $\left(J_{N}\right) I_{N}: U^{2} \rightarrow U$ is a (implication) coimplication.

Proof: Clearly, since $(J) I$ satisfies $(\mathbf{J 1}) \mathbf{I} 1$ and $N$ satisfies $\mathbf{N 1},\left(J_{N}\right) I_{N}$ satisfies (I1) $\mathbf{J 1}$ and so $\left(J_{N}\right) I_{N}$ is a (an implication) coimplication 
In [4] and [30], the notions of (co)implications require some extra properties, namely (J2 and J3) I2 and I3 and an analogous result of Proposition 7 is provided by considering these notions. The next proposition shows that the $N$-dual pair of implication-coimplication satisfies analogous properties and so the results in [4, Proposition 6] and [30, Proposition 3.1] follow.

Proposition 8 [37, Theorem 1] Let $N$ be a strong negation and I a fuzzy implication. Then I satisfies the property $\boldsymbol{I} i$ iff $I_{N}$ satisfies the property $\boldsymbol{J}$, for all $i$, $2 \leq i \leq 19$.

\section{Interval-valued (co)implications}

Since real numbers may be identified with degenerate intervals in the context of interval mathematics, the boundary conditions that must be satisfied by the classical fuzzy implications can be naturally extended to interval fuzzy degrees, whenever degenerate intervals are considered, see e.g. [22, 23] and [24].

Definition 9 The binary function $\mathbb{I}(\mathbb{J}): \mathbb{U}^{2} \rightarrow \mathbb{U}$ is called an interval fuzzy (co)implication iff it satisfies the boundary conditions given by

$\mathbb{I} 1: \mathbb{I}([1,1],[1,1])=\mathbb{I}([0,0],[1,1])=\mathbb{I}([0,0],[0,0])=$ $[1,1]$ and $\mathbb{I}([1,1],[0,0])=[0,0]$;

$\mathbb{J} 1: \mathbb{J}([1,1],[1,1])=\mathbb{J}([1,1],[0,0])=\mathbb{J}([0,0],[0,0])=$ $[0,0]$ and $\mathbb{J}([0,0],[1,1])=[1,1]$.

In Proposition $10, \mathbb{I}_{\mathbb{N}}\left(\mathbb{J}_{\mathbb{N}}\right)$ denotes the $\mathbb{N}$-dual interval coimplication (implication) of an interval fuzzy implication $\mathbb{I}$ (coimplication $\mathbb{J}$ ). Thus, interval fuzzy implications and interval fuzzy coimplications can also be studied based on dual notions.

Proposition 10 [36, Proposition 3] Let $\mathbb{N}$ be a strong interval fuzzy negation. If a function $(\mathbb{I}) \mathbb{I}: \mathbb{U}^{2} \rightarrow \mathbb{U}$ is an interval fuzzy (co)implication then $\left(\mathbb{J}_{\mathbb{N}}\right) \mathbb{I}_{\mathbb{N}}: \mathbb{U}^{2} \rightarrow \mathbb{U}$ is an interval fuzzy (implication) coimplication.

Canonical representability of interval-valued (co)implications is considered in the following.

Proposition 11 [36, Proposition 6] [7, Proposition 21] A fuzzy (co)implication $I(J): U^{2} \longrightarrow U$ satisfies the properties $\mathbf{I} 1$ (J1) and $\mathbf{I} 2(\mathbf{J} 2)$ iff the interval fuzzy (co)implication $\widehat{I}(\widehat{J})$ can be expressed as

$$
\begin{aligned}
& \widehat{I}(X, Y)=[I(\bar{X}, \underline{Y}), I(\underline{X}, \bar{Y})], \\
& \widehat{J}(X, Y)=[J(\bar{X}, \underline{Y}), J(\underline{X}, \bar{Y})],
\end{aligned}
$$

Some properties usually demanded from a fuzzy coimplication $J$ (implication $I$ ) then can also be naturally extended to an interval-based approach. This section considers an analysis under which conditions such properties, usually demanded from interval-valued (co)implications, are preserved by representable (co)implications which are generated from aggregation functions in $\mathrm{M}$ and a pair $\left(\left(I, I_{N}\right)\right)\left(I, I_{N}\right)$ of mutual dual (co)implications. For all $X, Y, Z \in \mathbb{U}$, the interval extension of properties considered in Section 2 are listed below:

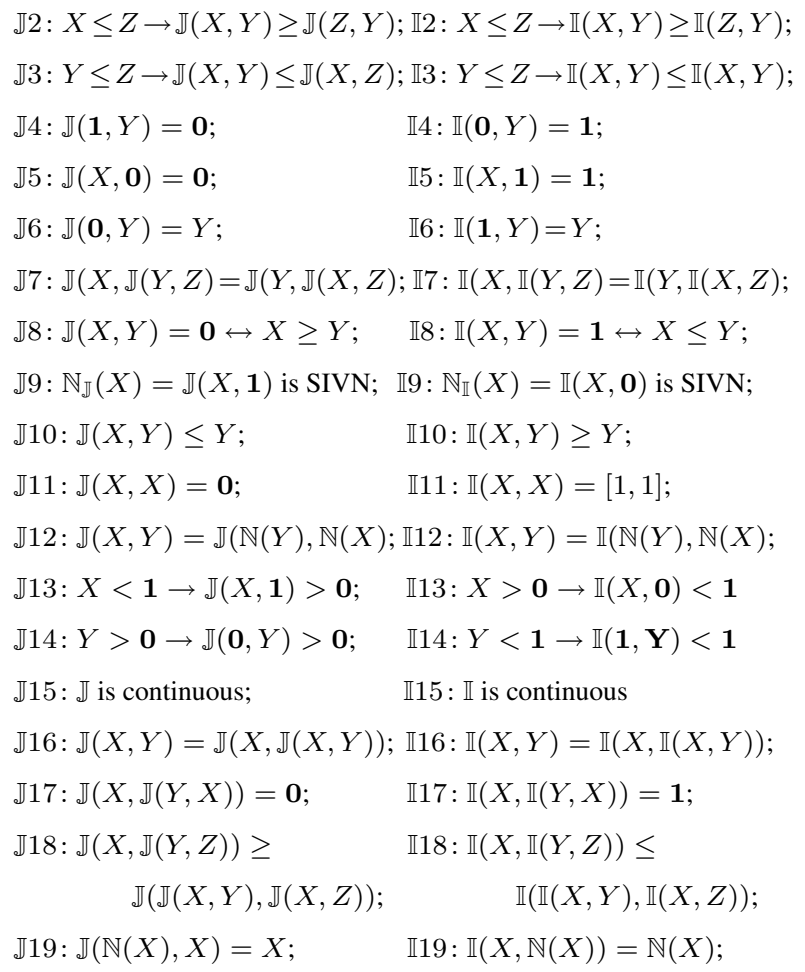

Proposition 12 [36, Theorem 2] Let $\mathbb{N}$ be a strong interval-valued negation and $\mathbb{I}$ an interval-valued implication. Then $\mathbb{I}$ satisfies the property $\mathbb{I} i$ iff $\mathbb{I}_{\mathbb{N}}$ satisfies the property $\mathbb{J} i$, for all $i, 2 \leq i \leq 13$.

The proposition 13 gives necessary and sufficient conditions to $\mathbb{I}$ be a $(\underline{\mathbb{I}}, \overline{\mathbb{I}})$-representable implication, whenever $\mathbb{I}(x, y)=l(\mathbb{I}([x, x],[y, y]))$ and $\overline{\mathbb{I}}=$ $r(\mathbb{I}([x, x],[y, y]))$. In a dual construction, $\mathbb{J}$ can also be a $(\underline{\mathbb{J}}, \overline{\mathbb{J}})$-representable coimplication when $\underline{\mathbb{J}}(x, y)=$ $l(\mathbb{J}([\bar{x}, x],[y, y]))$ and $\overline{\mathbb{J}}=r(\mathbb{J}([x, x],[y, y]))$.

Proposition 13 A function $\mathbb{I}(\mathbb{J}): \mathbb{U}^{2} \rightarrow \mathbb{U}$ is an inclusion monotonic interval (co)implication satisfying $\mathbb{I} 1, \mathbb{I} 2$ and $\mathbb{I} k$, for some $k=3, \ldots, 13$ iff there exist implications $\underline{\mathbb{I}}$ and $\overline{\mathbb{I}}$ with $\mathbb{I} \leq \overline{\mathbb{I}}$ (respectively, coimplications $\mathbb{J}$ and $\overline{\mathbb{J}}$ with $\mathbb{J} \leq \overline{\mathbb{J}}$ ) satisfying the properties $\mathbf{I} 1$ (J1), $\mathbf{I} 2$ $(\mathbf{J} 2)$ and $\mathbf{I} k(\mathbf{J} k)$, such that

$$
\begin{aligned}
\mathbb{I}(X, Y) & =[\underline{\mathbb{I}}(\bar{X}, \underline{Y}), \overline{\mathbb{I}}(\underline{X}, \bar{Y})], \\
\mathbb{J}(X, Y) & =[\underline{\mathbb{J}}(\bar{X}, \underline{Y}), \overline{\mathbb{J}}(\underline{X}, \bar{Y})],
\end{aligned}
$$

Proof: It follows from Theorems 17 and 19 in [7] and Propositions 10 and 12.

Notice that the results presented in Props. 12 and 13 can also be clearly extended to other properties, i.e. for $k=14, \ldots, 19$.

\section{Representable (co)implications generated from aggregation and dual operators}

An expression for interval-valued (co)implication generated from aggregation functions and mutual dual fuzzy 
(co)implications is introduced in this section, including the presentation of constraints assuring that such interval functions are well defined and may extend the canonical representation in the sense of [5].

Firstly, according to [10, Definition 2], an aggregation operator is a function $M: U^{2} \rightarrow U$ demanding:

A1 : $M(0,0)=0$ and $M(1,1)=1$;

A2 : If $x \leq z$ then $M(x, y) \leq M(z, y), \forall x, y, z \in U$;

A3 : $M(x, y)=M(y, x), \forall x, y \in U$;

Aggregation operators satisfying the idempotence property given below are called idempotent aggregation operators:

A4 : $M(x, x)=x, \forall x \in U$.

Moreover, when $M$ is an idempotent aggregation operator, then

$$
\wedge(x, y) \leq M(x, y) \leq \vee(x, y) \text { for all } x, y \in U .
$$

Definition 14 Let $I$ be an implication, $J$ be a coimplication, $N$ be a strong negation and $\left\{M_{i}: U^{2} \rightarrow\right.$ $U\}_{i \in\{1,2,3,4\}}$ be a subset of four idempotent aggregation functions. Then, $\mathbb{I}_{\left(I, I_{N}, M\right)}\left(\mathbb{J}_{\left(J, J_{N}, M\right)}\right): \mathbb{U}^{2} \rightarrow \mathbb{U}$ given by:

$$
\begin{aligned}
\mathbb{I}_{\left(I, I_{N}, M\right)}(X, Y) & =\left[\underline{\mathbb{I}}_{\left(I, I_{N}, M\right)}(X, Y), \overline{\mathbb{I}}_{\left(I, I_{N}, M\right)}(X, Y)\right] ; \\
\mathbb{J}_{\left(J, J_{N}, M\right)}(X, Y) & =\left[\mathbb{J}_{\left(J, J_{N}, M\right)}(X, Y), \overline{\mathbb{J}}_{\left(J, J_{N}, M\right)}(X, Y)\right] ;(10)
\end{aligned}
$$

and, such that the corresponding projection functions are defined by:

$$
\begin{array}{r}
\mathbb{I}_{\left(I, I_{N}, M\right)}(X, Y)=I\left(M_{1}(\underline{X}, \bar{X}), M_{2}(\underline{Y}, \bar{Y})\right), \\
\overline{\mathbb{I}}_{\left(I, I_{N}, M\right)}(X, Y)=N\left(I _ { N } \left(M_{3}(N(\bar{X}), N(\underline{X})),\right.\right. \\
\left.M_{4}(N(\bar{Y}), N(\underline{Y}))\right) ; \\
\underline{\mathbb{J}}_{\left(J, J_{N}, M\right)}(X, Y)=J\left(M_{3}(\underline{X}, \bar{X}), M_{4}(\underline{Y}, \bar{Y})\right), \\
\overline{\mathbb{J}}_{\left(J, J_{N}, M\right)}(X, Y)=N\left(J _ { N } \left(M_{1}(N(\bar{X}), N(\underline{X})),\right.\right. \\
\left.\left.M_{2}(N(\bar{Y}), N(\underline{Y}))\right)\right) ;
\end{array}
$$

is called $\left(\left(J, J_{N}, M\right)\right) \quad\left(I, I_{N}, M\right)$-representable (co)implications generated from aggregation functions in $M$ and by a pair $\left(\left(I, I_{N}\right)\right)\left(I, I_{N}\right)$ of mutual dual (co)implications.

The proposition below states the condition under which a triple $\left(\left(J, J_{N}, M\right)\right)\left(I, I_{N}, M\right)$ of fuzzy connectives on $U$ gives rise to a $\left(\left(J, J_{N}, M\right)\right)$ $\left(I, I_{N}, M\right)$-representable (co)implication.

Proposition 15 Consider $\left(\left(J, J_{N}\right)\right)\left(I, I_{N}\right)$ as a pair of $N-$ (co)implications satisfying (J2) I2 and (J3) I3. Let $\left\{M_{i}: U^{2} \rightarrow U\right\}_{i \in\{1,2,3,4\}}$ be a subset of four idempotente aggregation functions, such that, for all $X \in \mathbb{U}$,

$$
\begin{aligned}
& N\left(M_{1}(\underline{X}, \bar{X})\right) \leq M_{3}(N(\underline{X}), N(\bar{X})) ; \\
& N\left(M_{2}(\underline{X}, \bar{X})\right) \geq M_{4}(N(\underline{X}), N(\bar{X})) .
\end{aligned}
$$

Then, $\mathbb{I}_{\left(I, I_{N}, M\right)}\left(\mathbb{J}_{\left(J, J_{N}, M\right)}\right): \mathbb{U}^{2} \rightarrow \mathbb{U}$ given by Definition 14 is an interval (co)implication. In addition, $\mathbb{I}_{\left(I, I_{N}, M\right)}\left(\mathbb{J}_{\left(J, J_{N}, M\right)}\right)$ also satisfy $(\mathbf{J} 2) \mathbf{I} 2$ and $(\mathbf{J} 3) \mathbf{I} 3$.
Proof: We present the prove that $\mathbb{I}_{\left(I, I_{N}, M\right)}$ is an interval-valued implication in the sense of Definition 9. The other case can be proved in an analogous way.

If $N\left(M_{1}(\underline{X}, \bar{X})\right) \leq M_{3}(N(\underline{X}), N(\bar{X}))$ and $N\left(M_{2}(\underline{X}, \bar{X})\right) \geq M_{4}(N(\underline{X}), N(\bar{X}))$ and since $I_{N}$ verifies $\mathbf{J} 2$ and $\mathbf{J} 3$, then:

$N\left(I_{N}\left(M_{3}(N(\bar{X}), N(\underline{X})), M_{4}(N(\bar{Y}), N(\underline{Y}))\right)\right) \quad \geq$ $N\left(I_{N}\left(N\left(M_{1}(\underline{X}, \bar{X})\right), N\left(M_{2}(\underline{Y}, \bar{Y})\right)\right)\right)$ $I\left(M_{1}(\underline{X}, \bar{X}), M_{2}(\underline{Y}, \bar{Y})\right)$. Therefore, it is immediate that $\mathbb{I}_{\left(I, I_{N}, M\right)}$ is well defined, which means

$$
\overline{\mathbb{I}}_{\left(I, I_{N}, M\right)}(X, Y) \geq \underline{\mathbb{I}}_{\left(I, I_{N}, M\right)}(X, Y) .
$$

Since $\left\{M_{i}\right\}_{i \in\{1,2,3,4\}}$ is a subset of idempotent aggregation functions, by $\mathbf{A} 4 M_{1}(0,0)=M_{2}(0,0)=0$ and $M_{3}(1,1)=M_{4}(1,1)=1$. In addition, taking $\left(I_{N}\right) I$ as an (co)implication demanding the boundary conditions of Definition 9, by Eq (9) it holds that:

$\mathbb{I}_{\left(I, I_{N}, M\right)}([0,0],[0,0])=\left[I(0,0), N\left(I_{N}(1,1)\right)\right]=[1,1] ;$ $\mathbb{I}_{\left(I, I_{N}, M\right)}([0,0],[1,1])=\left[I(0,1), N\left(I_{N}(1,0)\right)\right]=[1,1] ;$ $\mathbb{I}_{\left(I, I_{N}, M\right)}([1,1],[1,1])=\left[I(1,1), N\left(I_{N}(0,0)\right)\right]=[1,1] ;$ $\mathbb{I}_{\left(I, I_{N}, M\right)}([1,1],[0,0])=\left[I(1,0), N\left(I_{N}(0,1)\right)\right]=[0,0]$.

Therefore $\mathbb{I}_{\left(I, I_{N}, M\right)}$ is an interval-valued implication. Analogously, by Eq (10), it can also be proved that $\mathbb{J}_{\left(J, J_{N}, M\right)}$ is an interval-valued coimplication. Finally, since aggregation operators are increasing in both components then $\mathbb{I}_{\left(I, I_{N}, M\right)}$ and $\left.\mathbb{J}_{\left(J, J_{N}, M\right)}\right)$ also satisfy $\mathbf{J} 2, \mathbf{J} 3$ and $\mathbf{I} 2, \mathbf{I} 3$, respectively.

In particular, by Eqs. (11) and (12), when $M_{1}$ and $M_{2}$ are the $N$-dual structure of $M_{3}$ and $M_{4}$, respectively, thus it holds that both $\mathbb{I}_{\left(I, I_{N}, M\right)}$ and $\mathbb{J}_{\left(J, J_{N}, M\right)}$ preserve degenerate intervals.

Corollary 16 Under the conditions stated in Proposition 15, if $\left(M_{1}, M_{3}\right)$ and $\left(M_{2}, M_{4}\right)$ are both pairs of mutual $N$-dual aggregation functions:

$$
\begin{aligned}
& N\left(M_{1}(\underline{X}, \bar{X})\right)=M_{3}(N(\underline{X}), N(\bar{X})) ; \\
& N\left(M_{2}(\underline{X}, \bar{X})\right)=M_{4}(N(\underline{X}), N(\bar{X})) ;
\end{aligned}
$$

then $\mathbb{I}_{\left(I, I_{N}, M\right)}$ and $\mathbb{J}_{\left(J, J_{N}, M\right)}$ are given by:

$$
\begin{aligned}
& \overline{\mathbb{I}}_{\left(I, I_{N}, M\right)}(X, Y)=\mathbb{I}_{\left(I, I_{N}, M\right)}(X, Y), \\
& \overline{\mathbb{J}}_{\left(J, J_{N}, M\right)}(X, Y)=\mathbb{I}_{\left(J, J_{N}, M\right)}(X, Y) .
\end{aligned}
$$

Proof: We present the proof of Eq. (15) since the other case is analogous. By duality states on Eq. (3),

$$
\begin{aligned}
\underline{\mathbb{I}}_{\left(I, I_{N}, M\right)}( & X, Y)=I\left(M_{1}(\underline{X}, \bar{X}), M_{2}(\underline{Y}, \bar{Y})\right), \\
= & I\left(N\left(M_{3}(N(\underline{X}), N(\bar{X}))\right),\right. \\
& \left.N\left(M_{4}(N(\underline{Y}), N(\bar{Y}))\right)\right), \\
= & N\left(I _ { N } \left(M_{3}(N(\underline{X}), N(\bar{X})),\right.\right. \\
& \left.\left.M_{4}(N(\underline{Y}), N(\bar{Y}))\right)\right)=\overline{\mathbb{I}}_{\left(I, I_{N}, M\right)}(X, Y)
\end{aligned}
$$

Therefore, $\mathbb{I}_{\left(I, I_{N}, M\right)}(X, Y)=\overline{\mathbb{I}}_{\left(I, I_{N}, M\right)}(X, Y)$

Corollary 17 Let $N$ be a strong fuzzy negation and (J) $I$ be a (co)implication such that $\left(\left(J, J_{N}, M\right)\right)$ $\left(I, I_{N}, M\right)$ is a triple of representants of an intervalvalued implication $\mathbb{I}_{\left(I, I_{N}, M\right)}$ generated by the subset of aggregation functions $\left\{M_{i}: U^{2} \rightarrow U\right\}_{i \in\{1,2,3,4\}}$. If 


$$
M_{1}=M_{3}=\vee \text { and } M_{2}=M_{4}=\wedge
$$

then $\left(\mathbb{I}_{\left(I, I_{N}, M\right)}\right) \mathbb{J}_{\left(J, J_{N}, M\right)}$ is the canonical representation of (J) I and (Eq. (10)) Eq. (9) can be reduced to (Eq. (6) ) Eq. (5).

Proof: We present the prove that $\mathbb{I}_{\left(I, I_{N}, M\right)}$ is the canonical representation of an implication $I$ according to Proposition 11. The other case can be proved analogously. If $M_{1}=M_{3}=\vee$ and $M_{2}=M_{4}=$ $\wedge$ then by Eq. (9) it holds that $\mathbb{I}_{\left(I, I_{N}, M\right)}(X, Y)=$ $\left[I(\underline{X}, \bar{Y}), N\left(I_{N}(N(\bar{X}), N(\underline{Y})] . \quad\right.\right.$ Thus, by Eq (9), $\mathbb{I}_{\left(I, I_{N}, M\right)}(X, Y)=[I(\underline{X}, \bar{Y}), I(\bar{X}, \underline{Y})]$.

Remark 1 In [12, 13] and [14], a formal treatment of interval-valued fuzzy sets and intuitionistic fuzzy sets are studied as special instances of the Goguen's $\mathcal{L}$-fuzzy sets, considering the bounded lattice $\left(\mathcal{L}^{*}, \leq_{\mathcal{L}^{*}}\right)$, whenever $\mathcal{L}^{*}=\left\{\left(x_{1}, y_{1}\right) \in[0,1]^{2}: x_{1}+y_{1} \leq 1\right\}$ and $\left(x_{1}, y_{1}\right) \leq_{\mathcal{L}^{*}}\left(x_{2}, y_{2}\right) \Leftrightarrow x_{1} \leq x_{2}$ and $y_{1} \geq y_{2}$, see [13, Definition 1].

Now, let $N$ be a strong fuzzy negation and $I$ be an implication such that $\left(I, I_{N}, M\right)$ is a triple of representants of an interval-valued implication $\mathbb{I}_{\left(I, I_{N}, M\right)}$ generated by the subset of aggregation functions $\left\{M_{i}: U^{2} \rightarrow\right.$ $U\}_{i \in\{1,2,3,4\}}$. Consider the isomorphism $h$ between $\mathbb{U}$ and $\mathcal{L}^{*}$ stating that:

$$
h(X) \mapsto(\underline{X}, 1-\bar{X}) ; \quad h^{-1}(x, y) \mapsto[x, 1-y]
$$

Thus, based on Proposition 15, $\mathbb{I}_{\left(I, I_{N}, M\right)}: \mathbb{U}^{2} \rightarrow \mathbb{U}$ is mapped into $\mathcal{I}_{\left(I, I_{N}, M\right)}: \mathcal{L}^{* 2} \rightarrow \mathcal{L}^{*}$, given by the expression in Eq. 17:

$$
\mathcal{I}_{\left(I, I_{N}, M\right)}=h \circ \mathbb{I}_{\left(I, I_{N}, M\right)} \circ\left(h^{-1} \times h^{-1}\right)
$$

Theorem 18 Consider $(J)$ I a (co)implication, a strong negation $N$ and $M=\left\{M_{i}: U^{2} \rightarrow U\right\}_{i \in\{1,2,3,4\}}$ as a subset of four idempotente aggregation functions. Let $\left(\mathbb{J}_{\left(J, J_{N}, M\right)}\right) \mathbb{I}_{\left(I, I_{N}\right)}$ be a $\left(\left(J, J_{N}, M\right)\right)$ $\left(I, I_{N}, M\right)$-representable (co)implication. Then $\mathbb{I}_{\left(I, I_{N}, M\right)}\left(\mathbb{J}_{\left(J, J_{N}, M\right)}\right)$ satisfies the property $\mathbb{I} k(\mathbb{J} k)$, for some $k \in\{1, \ldots, 5\}$ iff $I$ and $I_{N}\left(J\right.$ and $\left.J_{N}\right)$ satisfy $\mathbf{I} k$ and $\mathbf{J} i(\mathbf{J} k$ and $\mathbf{I} k)$, respectively.

Proof: We will prove that $\left(I, I_{N}, M\right)$-representable implication satisfies the property $\mathbb{I} i$, for $i \in\{1, \ldots 5\}$, if $I$ and $I_{N}$ satisfy $I i$ and $J i$, respectively.

II If $X \leq Z, \underline{X} \leq \underline{Z}$ and $\bar{X} \leq \bar{Y}$.

(i) By A2, A $3, M_{1}(\underline{X}, \bar{X}) \leq M_{1}(\underline{Z}, \bar{Z})$. So, by $\mathbf{I} 2$,

$$
\underline{\mathbb{I}}_{\left(I, I_{N}, M\right)}(X, Y) \geq \underline{\mathbb{I}}_{\left(I, I_{N}, M\right)}(Z, Y) .
$$

(ii) By $\mathbf{N} 2, N(\underline{X}) \geq N(\underline{Z})$ and $N(\bar{X}) \geq$ $N(\bar{Z})$. Thus, by $\mathbf{A} 3, M_{3}(N(\bar{X}), N(\underline{X})) \geq$ $M_{3}(N(\bar{Z}), N(\underline{Z}))$. Moreover, by $\mathbf{J} 2$ and $\mathbf{N} 2$,

$$
\overline{\mathbb{I}}_{\left(I, I_{N}, M\right)}(X, Y) \geq \underline{\mathbb{I}}_{\left(I, I_{N}, M\right)}(Z, Y) .
$$

Therefore, by Eqs. (18) and (19), it holds that $\mathbb{I}_{\left(I, I_{N}, M\right)}(X, Y) \geq \mathbb{I}_{\left(I, I_{N}, M\right)}(Z, Y)$, if $X \leq Z$.
I3 If $Y \leq Z, \underline{Y} \leq \underline{Z}$ and $\bar{Y} \leq \bar{Z}$. (i) By A3 and $M_{2}(\underline{Y}, \bar{Y}) \leq M_{2}(\underline{Z}, \bar{Z})$. So, $\mathbf{I} 3$

$$
\underline{I}_{\left(I, I_{N}, M\right)}(X, Y) \leq \underline{I}_{\left(I, I_{N}, M\right)}(X, Z) .
$$

(ii) By $\mathbf{N} 2, N(\underline{Y}) \geq N(\underline{Z})$ and $N(\bar{Y}) \geq$ $N(\bar{Z})$. Thus, by $\mathbf{A} 3, M_{4}(N(\bar{Y}), N(\underline{Y})) \geq$ $M_{4}(N(\bar{Z}), N(\underline{Z}))$. Moreover, by $\mathbf{J} 3$ and $\mathbf{N} 2$,

$$
\overline{\mathbb{I}}_{\left(I, I_{N}, M\right)}(X, Y) \leq \underline{\mathbb{I}}_{\left(I, I_{N}, M\right)}(Z, Y) .
$$

Therefore, by Eqs. (20) and (21), it is immediate that $\mathbb{I}_{\left(I, I_{N}, M\right)}(X, Y) \leq \mathbb{I}_{\left(I, I_{N}, M\right)}(Z, Y)$, if $Y \leq Z$.

$\mathbb{I} 4$ By $\mathbf{N} 1$ and $\mathbf{A} 1$, and since $I_{N}$ and $I$ satisfies $\mathbf{J} 4$ and I4, respectively, then $\mathbb{I}$ satisfy $\mathbb{I} 4$ :

$$
\begin{aligned}
\mathbb{I}_{I, I_{N}, M}([0,0], Y) & =I\left(M_{1}(0,0), M_{2}(\underline{Y}, \bar{Y})\right) \\
& =I\left(0, M_{2}(\underline{Y}, \bar{Y})\right)=1 ; \\
\overline{\mathbb{I}}_{I, I_{N}, M}([0,0], Y) & =N\left(I_{N}\left(1, M_{4}(N(\underline{Y}), N(\bar{Y}))\right)\right. \\
& =N(0)=1
\end{aligned}
$$

I5 Based on Properties N1 and A1 and since $J$ and $I$ satisfy $\mathbf{J} 5$ and $\mathbf{I} 5$, respectively, so $\mathbb{I}$ satisfies $\mathbb{I} 5$ :

$$
\begin{aligned}
\underline{\mathbb{I}}(X,[1,1]) & =I\left(M_{1}(\underline{X}, \bar{X}), M_{2}(1,1)\right) \\
& =I\left(M_{1}(\underline{X}, \bar{X}), 1\right)=1 ; \\
\overline{\mathbb{I}}(X,[1,1]) & =N\left(I _ { N } \left(M_{3}(N(\bar{X}, N(\underline{X})), 0)=1 .\right.\right.
\end{aligned}
$$

The converse is analogously done. Similarly, taking into account Proposition 12, one can obtain the related representable coimplication proof.

Hereinafter, from Propositions 19 to 32 , consider $\mathbb{I} \equiv$ $\mathbb{I}_{\left(I, I_{N}, M\right)}$ to simplify denotation. In order to analyze other properties, from $\mathbb{I} 6$ to $\mathbb{I} 19$, sufficient conditions are also presented, in addition to the constraint stated in Proposition 15.

Proposition 19 Let $I$ and $I_{N}$ be an implication and coimplication verifying $\mathbf{I} 6$ and $\mathbf{J} 6$, respectively. If $M_{1}=$ $M_{2}=\wedge$ then the related $\left(I, I_{N}, M\right)$-representable implication verifies $\mathbb{I} 6$.

Proof: By Properties N1 and A4 and since $I_{N}$ and $I$ satisfy $\mathbf{J} 6$ and $\mathbf{I} 6$, respectively, it holds that:

$$
\begin{aligned}
\underline{\mathbb{I}}([1,1], Y) & =I\left(1, M_{2}(\underline{Y}, \bar{Y})\right)=M_{2}(\underline{Y}, \bar{Y})=\underline{Y} \\
\overline{\mathbb{I}}([1,1], Y) & =N\left(I_{N}\left(0, M_{4}(N(\bar{Y}), N(\underline{Y}))\right)\right) \\
& =N\left(M_{4}(N(\bar{Y}), N(\underline{Y}))\right)=\bar{Y} .
\end{aligned}
$$

So, $\mathbb{I}([1,1], Y)=Y$ and $\mathbb{I}$ satisfies $\mathbb{I} 6$.

Proposition 20 Let $I$ and $I_{N}$ be an implication and $a$ coimplication verifying $\mathbf{I} 7$ and $\mathbf{J} 7$, respectively. Under the conditions of Corollary 16, then the related $\left(I, I_{N}, M\right)$-representable implication verifies $\mathbb{I} 7$.

Proof: Based on Eq. (15) states in Corollary 16,

$$
\underline{\mathbb{I}}(X, Z)=I\left(M_{1}(\underline{X}, \bar{X}), M_{2}(\underline{Z}, \bar{Z})\right)=\overline{\mathbb{I}}(X, Y) .
$$


Since $M_{1}$ and $M_{2}$ are idempotent aggregation functions, by $\mathbf{A} 4$, it holds that:

$$
\begin{aligned}
\mathbb{I}(Y, \mathbb{I}(X, Z)) & =I\left(M_{1}(\underline{Y}, \bar{Y}), M_{2}(\underline{\mathbb{I}}(X, Z), \overline{\mathbb{I}}(X, Z))\right) \\
& =I\left(M_{1}(\underline{Y}, \bar{Y}), M_{2}(\underline{\mathbb{I}}(X, Z), \overline{\mathbb{I}}(X, Z))\right) \\
& =I\left(M_{1}(\underline{Y}, \bar{Y}), \underline{\mathbb{I}}(X, Z)\right) \\
& =I\left(M_{1}(\underline{Y}, \bar{Y}), I\left(M_{1}(\underline{X}, \bar{X}), M_{2}(\underline{Z}, \bar{Z})\right)\right) \\
& =I\left(M_{1}(\underline{X}, \bar{X}), I\left(M_{1}(\underline{Y}, \bar{Y}), M_{2}(\underline{Z}, \bar{Z})\right)\right) \\
& =I\left(M_{1}(\underline{X}, \bar{X}), I(\underline{\mathbb{I}}(Y, Z))\right) \\
& =I\left(M_{1}(\underline{X}, \bar{X}), I\left(M_{2}(\underline{\mathbb{I}}(Y, Z), \overline{\mathbb{I}}(Y, Z))\right)\right) \\
& =\underline{I}(X, \mathbb{I}(Y, Z))
\end{aligned}
$$

So, $\mathbb{I}(Y,(X, Z))=\mathbb{I}(X,(Y, Z))$. Analogously, by Eq. (16), in Corollary 16, one can prove that $\overline{\mathbb{I}}(Y,(X, Z))=\overline{\mathbb{I}}(X,(Y, Z))$. Therefore, $\mathbb{I}$ satisfies $\mathbb{I} 7$.

Proposition 21 Let $I$ and $I_{N}$ be an implication and a coimplication verifying $\mathbf{I} 8$ and $\mathbf{J} 8$, respectively. If $M_{2} \geq M_{1}$ and $M_{4} \geq M_{3}$ then the related $\left(I, I_{N}, M\right)$-representable implication verifies $\mathbb{I} 8$.

Proof: If $X \leq Y$, by $\mathbf{A} 2, M_{1}(\underline{X}, \bar{X}) \leq$ $M_{1}(\underline{Y}, \bar{Y}) \leq M_{2}(\underline{Y}, \bar{Y})$ and $M_{3}(N(\bar{X}), N(\underline{X})) \leq$ $M_{3}(N(\bar{Y}), N(\underline{Y})) \leq M_{4}(N(\bar{Y}), N(\underline{Y}))$. In addition, since $M_{1}$ and $M_{2}$ are idempotent aggregation functions, by $\mathbf{A} 4$, it holds that $\mathbb{I}(X, Y)=[1,1]$ :

$$
\begin{gathered}
\underline{\mathbb{I}}(X, Y)=I\left(M_{1}(\underline{X}, \bar{X}), M_{2}(\underline{Y}, \bar{Y})\right)=1 \\
\overline{\mathbb{I}}(X, Y)=N\left(I _ { N } \left(\left(M_{3}(N(\underline{X}), N(\bar{X})),\right.\right.\right. \\
\\
\left.\left.\left.M_{4}(N(\underline{Y}), N(\bar{Y}))\right)\right)\right)=1
\end{gathered}
$$

So, II satisfies $\mathbb{I} 8$.

Proposition 22 Let $I$ and $I_{N}$ be an implication and $a$ coimplication verifying $\mathbf{I} 9$ and $\mathbf{J} 9$, respectively. If $M_{1}=$ $M_{3}=\wedge$ then the related $\left(I, I_{N}, M\right)$-representable implication verifies $\mathbb{I} 9$.

Proof: Taking $M_{1}=M_{3}=\wedge$ and $\mathbb{N}_{\mathbb{I}}(X)=$ $\mathbb{I}(X,[0,0])$. Since $N_{I}(N(\bar{X}))=I(N(\bar{X}, 0))=$ $N\left(I_{N}(\bar{X}, 1)\right)=N\left(N_{I_{N}}(\bar{X})\right)$, then it holds that

$$
\begin{aligned}
\underline{\mathbb{I}}(X,[0,0]) & =I\left(M_{1}(\underline{X}, \bar{X}), 0\right)=N_{I}(\underline{X}) ; \\
\overline{\mathbb{I}}(X,[0,0]) & \left.=N\left(I_{N}\left(M_{3}(N(\underline{X}), N(\bar{X})), 1\right)\right)\right) \\
& =N\left(I_{N}(N(\bar{X}), 1)\right)=N\left(N_{I_{N}}(N(\bar{X}))\right. \\
& =N\left(N_{I_{N}}(N(\bar{X}))\right)=N_{I}(\bar{X}) .
\end{aligned}
$$

So, $\mathbb{N}_{\mathbb{I}}(X)=\left[N_{I}(\bar{X}), N_{I}(\bar{X})\right]$. Moreover, $\mathbb{N}_{\mathbb{I}}$ is an IVSN, that means, $\mathbb{N}$ satisfies the next three properties:

$\mathbb{N} 1$ : By $\mathbf{N} 1, \mathbb{I}([0,0],[0,0])=\mathbb{N}_{\mathbb{I}}([0,0])=[1,1]$ and $\mathbb{I}([1,1],[0,0])=\mathbb{N}_{\mathbb{I}}([1,1])=[0,0]$;

$\mathbb{N} 2: \quad$ By $\mathbf{N} 2$, if $X \leq Y$ then $\mathbb{I}(X,[0,0])=$ $\left[N_{I}(\bar{X}), N_{I}(N(\bar{X}))\right] \geq\left[N_{I}(\bar{Y}), N_{I}(N(\bar{Y}))\right]=$ $\mathbb{I}(Y,[0,0])$;

$\mathbb{N} 3$ : By $\mathbf{N} 1, \mathbb{N}_{\mathbb{I}}\left(\mathbb{N}_{\mathbb{I}}(X)\right)=\mathbb{N}_{\mathbb{I}}\left(\left[N_{I}(\underline{\underline{X}}), N_{I}(\bar{X})\right]\right)=$ $\left[N_{I}\left(N_{I}(\underline{X})\right), N_{I}\left(N_{I}(\bar{X})\right)\right]=[\underline{X}, \bar{X}]=X$.
Therefore, $\mathbb{I}$ satisfies $\mathbb{I} 9$.

Proposition 23 Let $I$ and $I_{N}$ be an implication and a coimplication verifying $\mathbf{I} 10$ and $\mathbf{J} 10$, respectively. If $M_{2}=M_{4}=\wedge$ then the related $\left(I, I_{N}, M\right)$-representable implication verifies $\mathbb{I} 10$.

Proof: If $M_{2}=M_{4}=\wedge$, by $\mathbf{I} 10$ and $\mathbf{J} 10$, it holds that

$$
\begin{aligned}
\underline{\mathbb{I}}(X, Y)= & I\left(M_{1}(\underline{X}, \bar{X}), M_{2}(\underline{Y}, \bar{Y})\right) \geq M_{2}(\underline{Y}, \bar{Y})=\underline{Y} \\
\overline{\mathbb{I}}(X, Y)= & N\left(I _ { N } \left(M_{3}(N(\underline{X}), N(\bar{X})),\right.\right. \\
& \left.\left.M_{4}(N(\underline{Y}), N(\bar{Y}))\right)\right) \\
\geq & N\left(M_{4}(N(\underline{Y}), N(\bar{Y}))\right)=N(N(\bar{Y}))=\bar{Y} .
\end{aligned}
$$

So, $\mathbb{I}(X, X) \geq Y$. Therefore, $\mathbb{I}$ satisfies $\mathbb{I} 10$.

Proposition 24 Let $I$ and $I_{N}$ be an implication and a coimplication verifying $\mathbf{I} 11$ and $\mathbf{J} 11$, respectively. If $M_{1}=M_{2}$ and $M_{3}=M_{4}$ then the related $\left(I, I_{N}, M\right)$-representable implication verifies $\mathbb{I} 11$.

Proof: If $M_{1}=M_{2}$ and $M_{3}=M_{4}$, by $\mathbf{I} 10$ and $\mathbf{J} 10$, it holds that $\mathbb{I}$ satisfies $\mathbb{I} 11$ :

$$
\begin{aligned}
\underline{\mathbb{I}}(X, X)= & I\left(M_{1}(\underline{X}, \bar{X}), M_{1}(\underline{X}, \bar{X})\right)=1 \\
\overline{\mathbb{I}}(X, Y)= & N\left(I _ { N } \left(M_{3}(N(\underline{X}), N(\bar{X})),\right.\right. \\
& \left.\left.\left.M_{3}(N(\underline{X}), N(\bar{X}))\right)\right)=N(0)\right)=1 .
\end{aligned}
$$

So, $\mathbb{I}(X, X)=[\mathbb{I}(X, X), \overline{\mathbb{I}}(X, X)]=[1,1]$.

Proposition 25 Let $I$ and $I_{N}$ be an implication and $a$ coimplication verifying $\mathbf{I} 12$ and $\mathbf{J} 12$, respectively. If $N(Y)=X$ then the related $\left(I, I_{N}, M\right)$-representable implication verifies $\mathbb{1} 12$.

Proof: If $N(Y)=X$, by $\mathbf{I} 12$ and $\mathbf{J} 12$, it holds that

$$
\begin{aligned}
\underline{\mathbb{I}}(N(Y), N(X))= & I\left(M_{1}(N(\bar{Y}), N(\underline{Y})),\right. \\
& \left.M_{2}(N(\bar{X}), N(\underline{X}))\right) \\
= & I\left(M_{1}(\underline{X}, \bar{X}), M_{2}(\underline{Y}, \bar{Y})\right)=\mathbb{I}(X, Y) \\
\overline{\mathbb{I}}(N(Y), N(X))= & N\left(I_{N}\left(M_{3}(\underline{Y}, \bar{Y}), M_{4}(\underline{X}, \bar{X})\right)\right. \\
= & N\left(I _ { N } \left(M_{3}(N(\underline{X})), N(\bar{X}),\right.\right. \\
& M_{4}(N(\bar{Y}), N(\underline{Y}))=\overline{\mathbb{I}}(X, Y)
\end{aligned}
$$

So, $\mathbb{I}(N(Y), N(X))=\mathbb{I}(X, Y)$ and $\mathbb{I}$ satisfies $\mathbb{I} 12$.

Proposition 26 Let $I$ and $I_{N}$ be an implication and $a$ coimplication verifying $\mathbf{I} 12$ and $\mathbf{J} 12$, respectively. If $N(Y)=X$ then the related $\left(I, I_{N}, M\right)$-representable implication verifies $\mathbb{I} 13$.

Proof: Firstly, suppose that $X>[0,0]$, thus it follows that: $(i) M_{1}(\underline{X}, \bar{X})>M_{1}(0,0)=0$ and by $\mathbf{I} 13$, it holds that $I\left(M_{1}(\underline{X}, \bar{X}), 0\right)<1 ;(i i) N(X)<[1,1]$, then, by $\mathbf{N} 2, M_{3}(N(\bar{X}), N(\underline{X}))<M_{3}(1,1)=1$ and so, by $\mathbf{J} 13, I_{N}\left(M_{3}(N(\bar{X}), N(\underline{X})), 1\right)>0$ and one can conclude that $\left.N\left(I_{N}\left(M_{3}(N(\underline{X}), N(\bar{X})), 1\right)\right)\right)<$ $N(0)=1$. Based on that, it is immediate that

$$
\begin{aligned}
& \underline{I}(X,[0,0])=I\left(M_{1}(\underline{X}, \bar{X}), 0\right)<1 \\
& \left.\overline{\mathbb{I}}(X,[0,0])=N\left(I_{N}\left(M_{3}(N(\underline{X}), N(\bar{X})), 1\right)\right)\right)<1 ;
\end{aligned}
$$

So, $\mathbb{I}(X,[0,0])<[1,1]$ and $\mathbb{I}$ satisfies $\mathbb{I} 13$. 
Proposition 27 Let $I$ and $I_{N}$ be an implication and a coimplication verifying $\mathbf{I} 14$ and $\mathbf{J} 14$, respectively. If $N(Y)=X$ then the related $\left(I, I_{N}, M\right)$-representable implication verifies $\mathbb{I} 14$.

Proof: Firstly, suppose that $Y<[1,1]$, thus it follows that: $(i) M_{2}(\underline{Y}, \bar{Y})<M(1,1)=1$ and by $\mathbf{I} 14$, it holds that $I\left(1, M_{1}(\underline{Y}, \bar{Y})\right)<1$; $(i i) N(Y)<[1,1]$, then, by N2, $M_{4}(N(\bar{Y}), N(\underline{Y}))<M(1,1)=1$ and so, by $\mathbf{I} 13$, $I_{N}\left(M_{4}(N(\bar{Y}), N(\underline{Y})), 1\right)>0$, one can conclude that $N\left(I_{N}\left(M_{4}(N(\underline{Y}), N(\bar{Y})), 1\right)\right)<N(0)=1$. Based on that, it is immediate that

$$
\begin{aligned}
& \underline{\mathbb{I}}([1,1], Y)=I\left(1, M_{2}(\underline{Y}, \bar{Y})<1\right. \\
& \left.\overline{\mathbb{I}}([1,1], Y)=N\left(I_{N}\left(0, M_{4}(N(\underline{Y}), N(\bar{Y})), 1\right)\right)\right)<1 ;
\end{aligned}
$$

So, $\mathbb{I}([1,1], Y)<[1,1]$ and $\mathbb{I}$ satisfies $\mathbb{I} 14$.

Proposition 28 Let $I$ and $I_{N}$ be an implication and $a$ coimplication verifying $\mathbf{I} 15$ and $\mathbf{J} 15$, respectively. Their $\left(I, I_{N}, M\right)$-representable implication verifies $\mathbb{I} 15$.

Proof: Straightforward from the continuity of $I, I_{N}$. $\square$

Proposition 29 Let $I$ and $I_{N}$ be an implication and $a$ coimplication verifying $\mathbf{I} 16$ and $\mathbf{J} 16$, respectively. Under the conditions of Corollary 16, then the related $\left(I, I_{N}, M\right)$-representable implication verifies $\mathbb{I} 16$.

Proof: Based on Eq. (15) stated in Corollary 16,

$$
\underline{\mathbb{I}}(X, Y)=I\left(M_{1}(\underline{X}, \bar{X}), M_{2}(\underline{Y}, \bar{Y})\right)=\overline{\mathbb{I}}(X, Y) .
$$

Since $M_{1}$ and $M_{2}$ are idempotent aggregation functions, by $\mathbf{A} 4$, it holds that:

$$
\begin{aligned}
\mathbb{I}(X, & \mathbb{I}(X, Y))=I\left(M_{1}(\underline{X}, \bar{X}), I\left(M_{1}(\underline{X}, \bar{X}), M_{2}(\underline{Y}, \bar{Y})\right)\right) \\
\quad & \left.I\left(M_{1}(\underline{X}, \bar{X}), M_{2}(\underline{Y}, \bar{Y})\right)=\underline{\mathbb{I}}(X, Y)\right) ; \\
\overline{\mathbb{I}}(X, & \mathbb{I}(X, Y))=N\left(I _ { N } \left(M_{3}(N(\underline{X}), N(\bar{X})),\right.\right. \\
& \left.I_{N}\left(M_{3}(N(\bar{X}), N(\bar{X})), M_{4}(N(\bar{Y}), N(\underline{Y}))\right)\right) \\
& =N\left(I_{N}\left(M_{3}(N(\underline{X}), N(\bar{X})), M_{4}(N(\underline{Y}), N(\bar{Y}))\right)\right. \\
& =\overline{\mathbb{I}}(X, Y))
\end{aligned}
$$

So, $\mathbb{I}(X, \mathbb{I}(X, Y))=\mathbb{I}(X, Y)$.

Proposition 30 Let $I$ and $I_{N}$ be an implication and $a$ coimplication verifying $\mathbf{I} 17$ and $\mathbf{J} 17$, respectively. Under the conditions of Corollary 16, if $M_{1}=M_{2}$ then the related $\left(I, I_{N}, M\right)$-representable implication verifies $\mathbb{I} 17$.

Proof: Based on Eq. (15) states in Corollary 16,

$$
\underline{\mathbb{I}}(X, Y)=I\left(M_{1}(\underline{X}, \bar{X}), M_{2}(\underline{Y}, \bar{Y})\right)=\overline{\mathbb{I}}(X, Y) .
$$

Since $M_{1}$ and $M_{2}$ are idempotent aggregation functions, by $\mathbf{A} 4, \mathbf{I} 17$ and $\mathbf{J} 17$ it holds that:

$$
\begin{aligned}
& \mathbb{I}(X, \mathbb{I}(Y, X))=I\left(M_{1}(\underline{X}, \bar{X})\right. \\
& \left.\quad I\left(M_{1}(\underline{Y}, \bar{Y}), M_{2}(\underline{X}, \bar{X})\right)\right)=1 \\
& \overline{\mathbb{I}}(X, \mathbb{I}(Y, X))=N\left(I _ { N } \left(M_{3}(N(\underline{X}), N(\bar{X}))\right.\right. \\
& \left.\quad I_{N}\left(M_{3}(N(\bar{Y}), N(\bar{Y})), M_{4}(N(\bar{X}), N(\underline{X}))\right)\right) \\
& \left.\left.\left.\quad=\left(I\left(M_{1}(\underline{X}), \bar{X}\right), M_{1}(\underline{Y}), \bar{Y}\right), M_{2}(\underline{X}, \bar{X})\right)\right)\right)=1 . \\
& \text { So, } \mathbb{I}(X, \mathbb{I}(Y, X))=[1,1] .
\end{aligned}
$$

Proposition 31 Let $I$ and $I_{N}$ be an implication and a coimplication verifying $\mathbf{I} 18$ and $\mathbf{J} 18$, respectively. Under the conditions of Corollary 16, then the related $\left(I, I_{N}, M\right)$-representable implication verifies $\mathbb{I} 18$.

Proof: Based on Eq. (15) stated in Corollary 16,

$\underline{\mathbb{I}}(X, Y)=I\left(M_{1}(\underline{X}, \bar{X}), M_{2}(\underline{Y}, \bar{Y})\right)=\overline{\mathbb{I}}(X, Y)$, and $\underline{\mathbb{I}}(X, Z)=I\left(M_{1}(\underline{X}, \bar{X}), M_{2}(\underline{Z}, \bar{Z})\right)=\overline{\mathbb{I}}(X, Z) ;$

Since $M_{1}$ and $M_{2}$ are idempotent aggregation functions, by $\mathbf{A} 4, \mathbf{I} 18$ and $\mathbf{J} 18$ it holds that $\mathbb{I}$ verifies $\mathbb{I} 18$ :

$$
\begin{aligned}
\underline{I} & (\mathbb{I}(X, Y), \mathbb{I}(X, Z))=I\left(I \left(M_{1}(\underline{X}, \bar{X}), M_{2}(\underline{Y}, \bar{Y}),\right.\right. \\
& \left.I\left(M_{1}(\underline{X}, \bar{X}), M_{2}(\underline{Z}, \bar{Z})\right)\right) \\
\geq & I\left(M_{1}(\underline{X}, \bar{X}), I\left(M_{2}(\underline{Y}, \bar{Y}), M_{2}(\underline{Z}, \bar{Z})\right)\right)=\mathbb{I}(X, \mathbb{I}(Y, Z))
\end{aligned}
$$

Now, by Corollary $16, \mathbb{I}(X, \mathbb{I}(X, Y)=\overline{\mathbb{I}}(X, \mathbb{I}(X, Y)$. So, $\mathbb{I}(X, \mathbb{I}(Y, X))=\mathbb{I}(\mathbb{I}(X, Z), \mathbb{I}(Y, Z))$.

Proposition 32 Let $I$ and $I_{N}$ be an implication and $a$ coimplication verifying $\mathbf{I} 19$ and $\mathbf{J} 19$, respectively. If $M_{1}=M_{3}=\vee$ and $M_{2}=M_{4}=\wedge$, then the related $\left(I, I_{N}, M\right)$-representable implication verifies $\mathbb{I} 19$.

Proof: When $M_{1}=M_{3}=\vee$ and $M_{2}=M_{4}=\wedge$,

$$
\begin{aligned}
\underline{\mathbb{I}}(X, N(X)) & =I\left(M_{1}(\underline{X}, \bar{X}), M_{2}(N(\bar{X}), N(\underline{X}))\right) \\
& =I(\bar{X}, N(\bar{X}))=N(\bar{X}) \\
\overline{\mathbb{I}}(X, N(X)) & =N\left(I_{N}\left(M_{3}(N(\bar{X}), N(\underline{X})), M_{4}(\underline{X}, \bar{X})\right)\right) \\
& \left.=N\left(I_{N}(N(\underline{X}, \underline{X}))\right)=(N(\underline{X}))\right)
\end{aligned}
$$

Then, $\mathbb{I}(X, N(X))=\mathbb{N}(X)$ and $\mathbb{I}$ satisfies $\mathbb{I} 19$.

\section{Conclusion and Final Remarks}

Interval-valued fuzzy sets have been studied as a natural generalization of fuzzy sets modeling the uncertainty due to the lack of information in the definition of a membership function. Dealing with representable (co)implication generated from aggregation and dual operators, the paper introduces a generalization of the definition of a fuzzy implication operator introduced by Bustince et al. [10] as an important contribution. Based on that, we study the classes of dual-interval functions, called interval-valued (co)implications which can be obtained as representable (co)implications from aggregation operators and mutual dual fuzzy (co)implications. Thus, the main properties of the interval-valued (co)implications are investigated, showing the conditions under which the fuzzy (co)implications and aggregation operators should be satisfied to obtain the corresponding representable (co)implication and to preserve such properties. Our current work focusses on the study of two main topics:

(i) special classes of representable coimplications, such as $R-, S-, Q L-$ and $D$-coimplication classes in order to analyze the action of automorphisms and reductions on these classes;

(ii) study the connection between representable coimplications and interval-valued fuzzy coimplications starting from fuzzy connectives and $K$-operators, as conceived in [11].

Acknowledgments Thanks for the anonymous referees for their valuable comments and suggestions. 


\section{References}

[1] K. H. Atanassov. Intuitionistic Fuzzy Sets. Fuzzy Sets and Systems, 20:87-96, 1986.

[2] K. H. Atanassov. Intuitionistic Fuzzy Sets, Theory and Applications. Physica-Verlag, Heidelberg, 1999.

[3] M. Baczyǹski and B. Jayaram. (S,N)- and Rimplications: A state-of-the-art survey. Fuzzy Sets and Systems, 159:1836-1859, 2008.

[4] B. De Baets, Coimplicatiors, the forgotten connectives. Tatra Mountains Math. Publications, 12:229-240, 1997.

[5] B. Bedregal and A. Takahashi. The best interval representation of t-norms and automorphisms. Fuzzy Sets and Systems, 157(24):3220-3230, 2006.

[6] B. Bedregal and A. Takahashi. Interval valued versions of t-conorms, fuzzy negations and fuzzy implications. In Proc. of the IEEE Intl. Conf. on Fuzzy Systems, Vancouver, 2006, pages 553-559. IEEE, 2006.

[7] B. Bedregal and G. Dimuro and R. Santiago and R. Reiser. On interval fuzzy $S$-implications. Information Science, 180:1373-1389, 2010.

[8] B. Bedregal. On Interval Fuzzy Negations. Fuzzy Sets and Systems, 161:2290-2313, 2010.

[9] H. Bustince, P. Burillo, and F. Soria. Automorphism, negations and implication operators. Fuzzy Sets and Systems, 134:209-229, 2003.

[10] H. Bustince, E. Barrenechea, and V. Mohedano. Intuitionistic Fuzzy Implication Operators - an Expression and main Properties. Intl. Journal of Uncertainty Fuzziness and Knowledge-Based Systems, 12(3):387406, 2004.

[11] H. Bustince, E. Barrenechea, M. Pagola. Generation of Interval-Valued Fuzzy and Atanassov's Intuitionistic Fuzzy Connectives from Fuzzy Connectives and from $K_{\alpha}$ Operators: Laws for Conjunctions and Disjunctions, Amplitude. Intl. Journal of Uncertainty Fuzziness and Knowledge-Based Systems, 23:680-714, 2008.

[12] G. Cornelis, G. Deschrijver, and E. Kerre. On the Representation of Intuitionistic Fuzzy t-Norms and t-conorms. IEEE Transacs. on Fuzzy Systems, 12(1):45-61, 2004.

[13] G. Cornelis, G. Deschrijver, and E. E. Kerre. Implications in intuitionistic fuzzy and interval-valued fuzzy set theory: construction, classification and application. Intl. Journal of Approximate Reasoning, 35:55-95, 2004.

[14] G. Cornelis, G. Deschrijver, and E. E. Kerre. Advances and challenges in interval-valued fuzzy logic. Fuzzy Sets and Systems, 157:622-627, 2006.

[15] G. Deschrijver. A representation of t-norms in intervalvaluedL-fuzzy set theory.Fuzzy Sets and Systems, 159(13): $1597-1618,2008$

[16] G. Deschrijver, C. Cornelis, and E. E. Kerre. On the Representation of Intuitionistic Fuzzy t-Norms and t-Conorms. IEEE Transactions on Fuzzy Systems, 12(01):45-61, 2004

[17] G. Deschrijver, E. E. Kerre and Smets-Magrez. Axioms for intuitionistic fuzzy R-implicators. Intl. Journal of Uncertainty, Fuzziness and Knowledge-Based Systems, 13:453-464, 2005.

[18] D. Dubois and H. Prade. Random sets and fuzzy interval analysis. Fuzzy Sets and Systems, 12:87-101, 1991.

[19] J. Fodor. On fuzzy implication operators. Fuzzy Sets and Systems, 42:293-300, 1991.

[20] J. Fodor and M. Roubens. FuzzyPref.Modellingand Multicriteria Decision Support. Kluwer,Dordrecht, 1994.

[21] J. Fodor. Contrapositive symmetry of fuzzy implications. Fuzzy Sets and Systems, 69:141-156, 1995.
[22] I. Grattan-Guiness. Fuzzy membership mapped onto interval and many-valued quantities. Z. Math. Logik. Grundladen Math., 22:149-160, 1975.

[23] M. Gehrke, C. Walker, and E. Walker. Some comments on interval valued fuzzy sets. Intl. Journal of Intelligent Systems, 11:751-759, 1996.

[24] M. Gehrke, C. Walker and E. Walker, Topological and Algebraic Structures in Fuzzy Sets: A handbook of recent developments in the mathematics of fuzzy sets, Fuzzy logics arising from strict De Morgan systems. S. Rodabaugh and E.P. Klement Editors, Dordrecht:Kluwer Academic Publishers, 455-473, 2003.

[25] G. Gierz, K. Hofmann, K. Keimel, J. D. Lawson, M. Mislove, and D. Scott. Continuous Lattices and Domains. Cambridge Press, Cambridge, 2003.

[26] S. Gottwald. Set theory for fuzzy sets of higher level. Fuzzy Sets and Systems, 2(2):125-151, 1979.

[27] T. Hickey, Q. Ju, and M. Emdem. Interval arithmetic: from principles to implementation. Journal of the ACM, 48(5):1038-1068, 2001.

[28] E. P. Klement, R. Mesiar, and E. Pap. Triangular Norms. Kluwer, Dordrecht, 2000.

[29] G.J. Klir Uncertainty and Information: foundations of generalized information theory. NJ: JW \& Sons, 2000.

[30] L. Lin and Z. Xia, Intuitionistic fuzzy implication operators: Expressions and properties, Journal of Applied Mathematics and Computing, 22(3):325-338, 2006.

[31] P. Li and S. Fang, A survey on fuzzy relational equations, part I: classification and solvability, Fuzzy Optimization and Decision Making, 8:179-229, 2009.

[32] M. Mas, M. Monserrat and E. Trillas, A survey on fuzzy implication functions, IEEE Transactions on Fuzzy Systems 15(6): 1107-1121, 2007.

[33] K.C. Maes and B. De Baets, Commutativity and selfduality: Two tales of one equation. Intl. Journal of Approximate Reasoning, 50:189-199, 2008.

[34] J.M. Mendel. Uncertain Rule-Based Fuzzy Logic Systems: Introduction and New Directions. Prentice Hall, Upper Saddle River, 2001.

[35] R.E. Moore. Methods and Applications of Interval Analysis. SIAM, Philadelphia, 1979.

[36] R. Reiser, B. Bedregal and G. Reis. Interval Valued Fuzzy Coimplication. A. Dawar and R. Queiroz, editors, Logic, Language, Information and Computation, number 6188 in LNAI, pages 204-2017. Springer, Berlin, 2010.

[37] R. Reiser and B. Bedregal. Automorphisms acting on $N$ dual fuzzy functions: implications and coimplications. in Cong. Nac. de Matemática Aplicada e Computacional, 1 : 1-10. SBMAC, SP, 2010.

[38] D. Ruiz and J. Torrens, Residual implications and coimplications from idempotent uninorms. Kybernetika, 40(1), 21-38, 2004.

[39] R. Sambuc. Fonctions $\phi$-floues. Application l'aide au diagnostic en pathologie thyroidienne. $\mathrm{PhD}$ thesis, Univ. Marseille, Marseille, 1975.

[40] R. Santiago, B. Bedregal, and B. Acióly. Formal aspects of correctness and optimality of interval computations. Formal Aspects of Computing, 18(2):231-243, 2006.

[41] Y. Shi, D. Ruan and E. Kerre. On the characterizations of fuzzy implications satisfying $I(x, y)=I(x, I(x, y))$. Information Sciences, 177(145):2954-2970, 2007.

[42] L. A. Zadeh. Fuzzy sets. Information and Control, 8(3):338-353, 1965.

[43] L. A. Zadeh. The concept of a linguistic variable and its application to approximate reasoning - I. Information Sciences, 6:199-249, 1975. 